\title{
Modifiers for Medical Grade Polymeric Systems used in FDM 3D Printing - Short Review
}

\author{
Szarlej P, Kucińska Lipka J, Gubańska I and Janik H* \\ Department of Polymers Technology, Faculty of Chemistry, Poland \\ *Corresponding author: Janik H, Department of Polymers Technology, Faculty of Chemistry, 80233 Gdansk, Poland
}

\begin{tabular}{|c|c|}
\hline ARTICLE INFO & ABSTRACT \\
\hline & Abbreviations: FDM: Fused Deposition Modeling; AM: Additive Manufacturing; CT: \\
\hline Published: March 14, 2019 & Computed Tomography; MRI: Magnetic Resonance Imaging; PCL: Poly-E-Caprolactone; \\
\hline $\begin{array}{l}\text { Citation: Szarlej P, Kucińska Lipka } \\
\text { J, Gubańska I, Janik H. Modifiers for } \\
\text { Medical Grade Polymeric Systems used } \\
\text { in FDM 3D Printing - Short Review. } \\
\text { Biomed J Sci \& Tech Res 15(5)-2019. }\end{array}$ & $\begin{array}{l}\text { Cellulose; EVA: Ethylene Vinyl Acetate; HME: Hot Melt Extrusion; TCP: Tricalcium } \\
\text { Phosphate; TEC: Triethyl Citrate; HA: Hydroxyapatite; MMC: Microcrystalline Cellulose; } \\
\text { TPUs: Thermoplastic Polyurethanes; DDS: Drug Delivery Systems; FDM: Fused Deposition } \\
\text { Modeling; HMPCs: Human Mesenchymal Progenitor Cells; SEM: Scanning Electron } \\
\text { Microscopy; ECM: Extracellular Matrix, FFF: Fused Filament Fabrication }\end{array}$ \\
\hline
\end{tabular}
BJSTR. MS.ID.002778.

\section{Introduction}

Fused deposition modeling (FDM) is one of the additive manufacturing (AM) techniques, also known as a Fused Filament Fabrication (FFF). Additive manufacturing refers to the various types of three-dimensional printing methods, based on the gradual superimposition of subsequent layers of material, in order to obtain a three-dimensional object [1,2]. FDM is one of the most commonly used and inexpensive 3D printing technology in medicine and it is based on the layering of plasticized thermoplastic polymeric material on a mobile platform through the miniature nozzle $[3,4]$. The material is supplied in the form of a thin wire of constant diameter, so-called filament. FDM technology also allows the use of CAD models and images obtained from computed tomography (CT) or magnetic resonance (MRI) [5,6]. The following biomedical polymers are considered to be used for medical grade filaments production for FDM technology: poly- $\varepsilon$-caprolactone (PCL) [7], polylactide (PLA) [8], polyvinyl alcohol (PVA) [9], hydroxypropyl cellulose (HPC) [10], ethyl cellulose (EC) [11], ethylene vinyl acetate (EVA) [11] or thermoplastic polyurethanes (TPUs) [12,13]. Furthermore, polymeric systems designed as filaments for 3D printing can be easily modified with many inorganic and organic compounds through the hot melt extrusion (HME).

For the improvement of the mechanical, thermal, rheological and viscoelastic properties of filaments, fillers and plasticizers can be applicated $[14,15]$. Low-molecular-weight plasticizers belong to the group of the most commonly used compounds for changing properties of polymers. They extend the free space between polymer chains, what has positive effect on the material processing. The most popular fillers and plasticizers include tricalcium phosphate (TCP) [16,17], triethyl citrate (TEC) [15], hydroxyapatite (HA) [18], mannitol [19] or microcrystalline cellulose (MMC) [20]. Moreover, filaments can be modified with lubricants (e.g. glycerol) to solve the problem of blocking the material inside the nozzle [15,21-23]. Polymeric filaments filled with biologically active compounds can be applied for producing of tissue scaffolds characterized by enhanced biocompatibility and also for printing some polymeric drug delivery systems (DDS) such as tablets, patches or intrauterine systems [23-27]. The most frequently tested pharmaceutical ingredients for the FDM printed drug delivery systems include prednisolone, salicic acid, paracetamol, acetaminophen, theophylline and indomethacin [23]. DDS show the ability to controlled and directed release of mentioned active agents by changing their pharmacokinetic and pharmacodynamic properties, as well as affecting their biodistribution, ability to cross the biological barrier and also resistance against metabolic reactions. The main advantage of using controlled release systems is to keep the concentration of the active compounds in the body in a safe therapeutic range, thus limiting the occurrence of side effects resulting from its use. An additional 
benefit of using DDS is a definitely longer persistence of the active substance in the body $[23,28,29]$.

\section{Characterization of the Most Popular Modifiers}

Hydroxyapatite (HA) is an excellent example of polymers modifier, which is frequently chosen inorganic filler for fabricating 3D scaffolds designed to rebuild bone tissue. Over $70 \%$ of inorganic composition of the bone mainly consists of hydroxyapatite, which makes it suitable component for promoting bone tissue regeneration [30]. Furthermore, it has been proven that hydroxyapatite has osteoconductive and osteoinductive properties, that result in a good cellular and tissue responses, while conducting in vitro and in vivo tests [31,32]. In biomedical constructs printed in FDM technology, hydroxyapatite generally occurs in compositions with PLA $[33,34]$ and PCL $[35,36]$. Moreover, hydroxyapatite has found application as a modifier of filaments made of polyamide (PA) [37] and also of PLA/PCL blends of different molar ratio [38].

Another usually used modifier of polymers for medical application is tricalcium phosphate (TCP). It is an inorganic compound characterized by chemical composition and properties similar to HA. It was reported that all commercially available calcium phosphate ingredients, including TCP, enhances the bioactivity, osteoconducitivity, osteoinductivity of printed constructs and also increases the formation of bone interface through the composite scaffolds [39].

The literature provides examples of TCP applications as a modifier in the process of extruding filaments made of PCL $[17,40]$, PLA [41] and polypropylene (PP) [40-42]. It was also observed that TCP in compositions with poly( $\alpha$-hydroxy acids) improves their mechanical properties and biocompatibility. TCP also has an ability to buffer acid degradation products of scaffold matrix made of poly( $\alpha$-hydroxy acids), which allows to avoid inflammatory effects of tissues [43]. More examples of particular modifiers will be provided in the extended review.

\section{Examples of Modified Filaments and Printed Products}

In 2016 Corcione et al. [18] evaluated the novel, solvent-free method of manufacturing PLA and also PLA/HA composite filaments containing $5 \%$ of HA. At the first step, PLA pellets were mixed with HA powder in a Rotomoulding machine, at a temperature close to the melting point of PLA, to obtain homogenous dispersion of ceramic compound in the polymer. Afterwards, coated pellets were processed into the functional filaments by hot melt extrusion technique using a twin screw extruder. Obtained filaments were characterized with a diameter of $1,75 \mathrm{~mm}$. PLA/HA composite filaments showed slightly increased flexural modulus in comparison to PLA reference filaments. Furthermore, SEM and EDX analyses confirmed the homogenous distribution of HA within the PLA matrix of the modified filaments. A successful print attempt of porous maxillary sinus ultimately affirmed the usefulness of the filaments obtained from PLA and HA [18].
In 2003 Hutchmacher et al. [32] also reported the possibility to fabricate three-dimensional, porous and bioresorbable PCL and PCL/HA (90:10) frameworks combined with a fibrynogen hydrogel containing human mesenchymal progenitor cells (hMPCs).

Scaffolds were prepared by fused deposition modeling (FDM) in order to obtain fully interconnected and honeycomb pore architecture. Each scaffold was characterized by lay-down pattern of $0 / 60 / 120^{\circ}$ and a porosity equal $65 \%$. The results of scanning electron microscopy (SEM) and confocal laser microscopy (CLM) indicated the adhesion of hMPCs onto the both types of fibers and bridging the pores of both constructs through the formation of extracellular matrix (ECM) after 2 weeks of cultivating. 3 weeks from the beginning of culture, both kinds of scaffolds revealed architecture completely filled with cells and extracellular matrix (ECM), what was proved by SEM and phase-contrast microscopy (PCM). Moreover, a significant quantity of cells exhibited activity throughout the entire cultivating period and imaging data revealed no differences in proliferation patterns between PCL and PCL- HA constructs. Metabolic assays exhibited, that hMPCs revealed the greatest differentiation along osteogenic, chondrogenic and adipogenic lineages through both types of scaffolds [32].

The next example of modified filament was investigated by Kalita et al. [42]. They obtained biocompatible PP/TCP composite material and processed it into porous scaffolds for hard tissue engineering. PP and TCP were compounded together with processing aids such as hard paraffin (lubricant) and vegetable oil (plasticizer), using torque rheometer for $30 \mathrm{~min}$ at $170^{\circ} \mathrm{C}$. Then, a single screw extruder was used to produce the filament of diameter $1,78 \mathrm{~mm}$. The final composition contained $20,5 \%$ of TCP. Obtained TCP- modified filament was used to print tissue scaffolds of different internal architecture and also compared with constructs made of neat PP and also PP/processing aids filaments. PP/TCP compositions were characterized by the highest elastic modulus. Biological assays confirmed that manufactured scaffolds were not toxic for modified human osteoblast cell-line (HOB). Furthermore, during the first 2 weeks of in vitro testing the excellent cell growth was observed [42].

\section{Conclusion}

In summary, it was found that FDM 3D printing technology could find a niche in the wide range of biomedical applications. Unfortunately, the quantity of polymeric biomaterials suitable to processing into filaments is still limited. The most commonly considered biomaterials for producing medical constructs such as bone grafts, soft tissue scaffolds or another DDS include PCL, PLA, PVA, HPC, EVA copolymer, EC and TPUs. These materials should exhibit appropriate properties depending on the intended application, so to improve their mechanical and biological characteristics, numerous modifiers such as TCP, HA, TEC, MMC and also wide range of pharmaceuticals may be used. The description of the modifiers in the point 2 and examples of filaments and printed constructs in- 
cluded in the point 3, allows to consider that the mentioned modifiers are promising compounds for producing filaments with increased biocompatibility and also mechanical, thermal, rheological and viscoelastic properties of filaments.

\section{References}

1. Tappa K, Jammalamadaka U (2018) Novel biomaterials used in medical 3D printing techniques. J Funct Biomater 7: 9(1).

2. Vanderburgh J, Sterling JA, Guelcher SA (2017) 3D Printing of Tissue Engineered Constructs for In Vitro Modeling of Disease Progression and Drug Screening. Ann Biomed Eng 45(1): 164-179.

3. Negi S, Dhiman S, Sharma RK (2017) Basics and applications of rapid prototyping medical models. Rapid Prototyp J 20(3): 256-267.

4. Bose S, Vahabzadeh S, Bandyopadhyay A (2013) Bone tissue engineering using 3D printing. Mater Today 16(12): 496-504.

5. Ligon SC, Liska R, Stampfl J, Gurr M, Mülhaupt R, et al. (2017) Polymers for 3D Printing and Customized Additive Manufacturing. Chem Rev 117(15): 10212-10290.

6. An J, Teoh JEM, Suntornnond R, Chua Ck (2015) Design and 3D Printing of Scaffolds and Tissues. Engineering 1(2): 261-268.

7. Korpela J, Kokkari A, Korhonen H, Malin M, Narhi T, et al. (2013) Biodegradable and bioactive porous scaffold structures prepared using fused deposition modeling. J Biomed Mater Res - Part B Appl Biomater 101(4): 610-619.

8. Luzuriaga MA, Berry DR, Reagan JC, Smaldone RA, Jeremiah J, et al. (2018) Biodegradable 3D Printed Polymer Microneedles for Transdermal Drug Delivery, p. 1-8.

9. Esposito Corcione C, Gervaso F, Scalera F, et al. (2017) 3D printing of hydroxyapatite polymer-based composites for bone tissue engineering. J Polym Eng 37(8): 741-746.

10. Chai X, Chai H, Wang X (2017) Fused deposition modeling (FDM) 3D printed tablets for intragastric floating delivery of domperidone. Sci Rep $7(1): 1-9$

11. Melocchi A, Parietti F, Maroni A, Foppoli A, Gazzaniga A, et al. (2016) Hot-melt extruded fi laments based on pharmaceutical grade polymers for 3D printing by fused deposition modeling. Int J Pharm 509(1-2): 255-263.

12. Przybytek A, Janik H (2016) Application in 3D printing $4: 32-39$.

13. Verstraete G, Samaro A, Grymonpré W (2018) 3D printing of high drug loaded dosage forms using thermoplastic polyurethanes. Int J Pharm 536(1): 318-325.

14. Konta A, García Piña M, Serrano D (2017) Personalised 3D Printed Medicines: Which Techniques and Polymers Are More Successful? Bioengineering 4(4): 79.

15. Sadia M, Sosnicka A, Arafat B (2016) Adaptation of pharmaceutical excipients to FDM 3D printing for the fabrication of patient-tailored immediate release tablets. Int J Pharm 513(1-2): 659-668.

16. Lei Y, Rai B, Ho KH, Teoh SH (2007) In vitro degradation of novel bioactive polycaprolactone-20\% tricalcium phosphate composite scaffolds for bone engineering 27: 293-298.

17. Rai B, Teoh SH, Hutmacher DW, Cao T, Ho KH, et al. (2005) Novel PCLbased honeycomb scaffolds as drug delivery systems for rhBMP-2. Biomaterials 26(17): 3739-3748.

18. Corcione CE, Gervaso F, Scalera F, Montagna F (2017) 3D printing of hydroxyapatite polymer-based composites for bone tissue engineering, p. 1-6.

19. Gioumouxouzis CI, Katsamenis OL, Bouropoulos N, Fatouros DG (2017) 3D printed oral solid dosage forms containing hydrochlorothiazide for controlled drug delivery. J Drug Deliv Sci Technol 40: 164-171.
20. Beck RCR, Chaves PS, Goyanes A (2017) 3D printed tablets loaded with polymeric nanocapsules: An innovative approach to produce customized drug delivery systems. Int J Pharm 528(1-2): 268-279.

21. Okwuosa TC, Stefaniak D, Arafat B, Isreb A, Wan KW, et al. (2016) A Lower Temperature FDM 3D Printing for the Manufacture of PatientSpecific Immediate Release Tablets. Pharm Res 33(11): 2704-2712.

22. Madan S, Madan S (2012) Hot melt extrusion and its pharmaceutical applications. 7(2): 123-133.

23. Cunha-Filho M, Araújo MR, Gelfuso GM, Gratieri T (2017) FDM 3D printing of modified drug-delivery systems using hot melt extrusion: A new approach for individualized therapy. Ther Deliv 8(11): 957-966.

24. Kotlarz M, Jordan R, Wegener E (2018) One step 3D printing of surface functionalized composite scaffolds for tissue engineering applications. Acta Bioeng Biomech 20(2): 35-45.

25. Genina N, Holländer J, Jukarainen H, Mäkilä E, Salonen J, et al. (2016) Ethylene vinyl acetate (EVA) as a new drug carrier for 3D printed medical drug delivery devices. Eur J Pharm Sci 90: 53-63.

26. Economidou SN, Lamprou DA, Douroumis D (2018) 3D printing applications for transdermal drug delivery. Int J Pharm 544(2): 415-424.

27. Systems DD (2018) International Journal of Drug Development and Application of 3D Printing Technology in the Development of Novel 18 776(1): 1-8.

28. Vilar G, Tulla puche J, Albericio F (2018) Polymers and Drug Delivery Systems, p. 1-28.

29. Liechty WB, Kryscio DR, Slaughter B V, Peppas NA (2010) Polymers for Drug Delivery Systems.

30. Id BH, Caetano G, Vyas C, Blaker JJ (2018) Polymer-Ceramic Composite Scaffolds : The Effect of Hydroxyapatite and $\beta$-tri-Calcium Phosphate 11: $1-20$.

31. Michel J, Penna M, Kochen J, Cheung H (2015) Recent Advances in Hydroxyapatite Scaffolds Containing Mesenchymal Stem Cells.

32. Sultana T, Rana M, Akhtar N, Hasan Z, Talukder AH (2017) Preparation and physicochemical characterization of nano- hydroxyapatite based $3 \mathrm{~d}$ porous scaffold for biomedical application 3(3): 384-389.

33. Endres M, Hutmacher DW, Salgado AJ (2003) Osteogenic Induction of Human Bone Marrow-Derived Mesenchymal Progenitor Cells in Novel Synthetic Polymer-Hydrogel Matrices. Tissue Eng 9(4): 689-702.

34. Corcione CE, Gervaso F, Madaghiele M, Sannino A, Licciulli A (2018) Author's Accepted Manuscript. Ceram Int.

35. Sousa FCG De, Evans JRG (2003) Sintered Hydroxyapatite Latticework for Bone Substitute 19: 517-519.

36. Phosphate T, Matrix B, Nyberg E, Rindone A, Dorafshar A (2017) Comparison of 3D-Printed Poly- e -Caprolactone Scaffolds 23(3): 503514.

37. Dudek P (2013) Agh university of science and technology, faculty of mechanical engineering and robotics. kraków, Poland, p. 2-5.

38. Wu J, Chen N, Bai F, Wang Q (2017) Preparation of Poly ( vinyl alcohol )/ Poly ( lactic acid )/ Hydroxyapatite Bioactive Nanocomposites for Fused Deposition Modeling, p. 1-11.

39. Legeros RZ (2002) Properties of Osteoconductive Biomaterials : Calcium Phosphates 395: 81-98.

40. Rai B, Teoh SH, Ho KH (2004) The effect of rhBMP-2 on canine osteoblasts seeded onto 3D bioactive polycaprolactone scaffolds. Biomaterials 25(24): 5499-5506.

41. Drummer D, Cifuentes Cuéllar S, Rietzel D (2012) Suitability of PLA/TCP for fused deposition modeling. Rapid Prototyp J 18(6): 500-507.

42. Kalita SJ, Bose S, Hosick HL, Bandyopadhyay A (2003) Development of controlled porosity polymer-ceramic composite scaffolds via fused deposition modeling. Mater Sci Eng C 23(5): 611-620. 
43. Li L, Xiong Z, Yonganian Y, Yunyu H, Renji Z, Shenguo W (2007) Porous morphology, porosity, mechanical properties of poly(a-hydroxy acid)-

\section{ISSN: 2574-1241}

DOI: 10.26717/BJSTR.2019.15.002778

Janik H. Biomed J Sci \& Tech Res

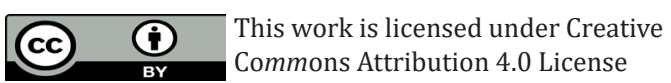

Submission Link: https://biomedres.us/submit-manuscript.php tricalcium phosphate composite scaffolds fabricated by low-temperature deposition. J Biomed Mater Res Part A pp. 618-629.

$\begin{array}{ll}\text { BIOMEDICAL } & \text { Assets of Publishing with us } \\ \text { RESEARCHES } & \text { - Global archiving of articles } \\ & \text { - Immediate, unrestricted online access } \\ & \text { - Rigorous Peer Review Process } \\ \end{array}$

